\title{
Les modalités d'alimentation des troupeaux ovins en steppe algérienne, région de M'Sila : pratiques et tendances
}

\author{
Ilyes Hadbaoui ${ }^{1,2, *}$, Abdelhakim Senoussi ${ }^{1}$ et Johann Huguenin ${ }^{3,4}$ \\ ${ }^{1}$ Laboratoire bioressources sahariennes : préservation et valorisation, Université Kasdi Merbah, Ouargla, Algérie \\ ${ }^{2}$ Centre de recherche scientifique et technique sur les régions arides (CRSTRA), Station Taouiala, Biskra, Algérie \\ ${ }^{3}$ Cirad, UMR SELMET, 34398 Montpellier, France \\ ${ }^{4}$ SELMET, Univ Montpellier, Cirad, INRA, Montpellier SupAgro, Montpellier, France
}

\begin{abstract}
Résumé - L'objectif de cet article est d'identifier les modalités d'alimentation des troupeaux ovins des steppes de la région de M'Sila en Algérie. Cette région présente un fort potentiel en matière d'élevage ovin sur ses parcours steppiques, qui ont connu depuis le siècle dernier des perturbations d'origine biophysique et anthropique altérant les surfaces, les bioressources et la productivité. En conséquence, les éleveurs ovins se voient contraints de chercher des alternatives pour alimenter leurs animaux. Afin d'apprécier les évolutions adoptées dans les élevages, nous avons retenu un échantillon raisonné de 100 éleveurs et agroéleveurs, interviewés avec l'aide de "personnes ressources locales» dont le rôle est de faciliter le contact. L'échantillonnage réalisé permet d'avoir une bonne représentation des différents systèmes d'élevage ovin rencontrés dans la région d'étude. L'interprétation des informations obtenues met en exergue trois modalités d'alimentation des troupeaux ovins. En alternative à l'utilisation massive des aliments concentrés dans la ration, l'intégration des cultures fourragères dans la conduite alimentaire des troupeaux ovins représente une nouvelle pratique pour une catégorie d'agroéleveurs. Trois types de cultures sont mis en place pour l'affouragement en vert: l'orge, l'avoine et la luzerne. Ces tendances apparaissent comme des solutions curatives et incomplètes face au manque de ressources pastorales. Néanmoins, elles peuvent inspirer des pistes de réflexions sur des solutions possibles au manque de ressources fourragères en milieu steppique en réduisant la charge animale sur les parcours.
\end{abstract}

Mots clés : parcours / élevage ovin / aliment alternatif / culture fourragère / steppe

\begin{abstract}
Feeding modalities of sheep flocks in the Algerian steppe, region of M'Sila: practices and trends. The objective of this article is to identify feeding modalities of sheep flocks in the steppes of the region of M'Sila in Algeria. This region has a strong potential for sheep farming on its steppe rangelands, which have experienced since the last century biophysical and anthropogenic disturbances altering lands, bio-resources and productivity. As a result, sheep farmers are forced to look for alternative solutions to feed their animals. In order to assess the changes adopted in the farms, we selected a reasoned sample of 100 breeders and agro-breeders, interviewed with the help of "local resource persons" whose role is to facilitate the contact. The sampling carried out provides a good representation of the different sheep breeding systems encountered in the study area. The interpretation of the information obtained highlights three ways of feeding sheep flocks. As an alternative to the massive use of concentrates in the diet, the integration of forage crops in the feeding of sheep flocks represents a new practice for a category of agropastoralists. Three types of crops are used for green feeding: barley, oats and alfalfa. These trends appear as curative and incomplete solutions to the lack of pastoral resources. Nevertheless, they can inspire reflections on possible solutions to the lack of fodder resources in steppe environments by reducing the stocking rate on rangelands.
\end{abstract}

Keywords: rangeland / sheep breeding / alternative feed / feed crop / steppe

\footnotetext{
* Auteur de correspondance : hadbaouililyes@yahoo.fr
} 


\section{Introduction}

Le territoire steppique algérien couvre 20 millions d'hectares, dont 13 millions d'hectares de parcours. Sa vocation ancestrale était l'élevage extensif d'ovins, de caprins et de dromadaires, complété par la culture épisodique de céréales (Aïdoud et al., 2006). Toutefois, les parcours naturels steppiques subissent depuis 50 ans une régression très sévère de leur surface et de leur productivité (Abbas, 2004). Aïdoud et al. (2006) ont signalé un déclin significatif de la productivité pastorale au cours des 40 dernières années; globalement, elle est inférieure à 50 unités fourragères (UF)/ha dans la majeure partie de la steppe. De plus, le cheptel ovin algérien, dont la plus grande partie est cantonnée dans la zone steppique, a connu un fort accroissement (Senoussi et al., 2011). Il est passé de 5 millions de têtes à l'aube de l'Indépendance (en 1962) à plus de 28 millions en 2017 (FAO, 2017), dont 60\% (17 millions de têtes) demeurent en zone steppique. Face à la diminution des ressources pastorales, le recours systématique à la complémentation par des aliments concentrés est devenu une pratique courante pour tous les éleveurs de la steppe (Bourbouze, 2000). Néanmoins, la complémentation par des aliments concentrés présente des risques liés à la dépendance aux marchés des aliments du bétail, dont les prix fluctuent en fonction des conditions climatiques et géopolitiques.

$\mathrm{Au}$ vu des conditions actuelles - diminution fourragère pastorale des parcours steppiques et instabilité des marchés d'aliments du bétail-, il est opportun de s'interroger sur les ressources alimentaires utilisées par les éleveurs pour nourrir leurs troupeaux. Pour répondre à cette interrogation, l'hypothèse émise est que les éleveurs introduisent de nouvelles ressources fourragères dans la ration alimentaire des ovins. L'objectif de la présente étude est d'identifier les différentes ressources fourragères ou alimentaires exploitées, ainsi que leurs modalités d'usage. Une approche de modélisation des rations permet une représentation graphique des profils alimentaires existants. Par ailleurs, l'étude apporte des éléments sur la conduite alimentaire, qui peuvent renforcer la résilience des systèmes d'élevage. Elle constitue, par conséquent, un apport pour les programmes de développement de l'élevage ovin steppique.

\section{Matériel et méthodes}

\subsection{Méthodologie}

La méthodologie utilisée repose sur des enquêtes renseignées lors d'un passage unique auprès d'éleveurs, choisis de façon à avoir un maximum de diversités des systèmes d'élevage ovin existants. Il existe deux grands systèmes d'élevage ovin recensés dans la région d'étude: les systèmes agropastoraux, les plus répandus, où l'association agriculture-élevage constitue le cœur de la gestion de l'exploitation; les systèmes pastoraux, où les éleveurs pratiquent principalement l'élevage (Senoussi et al., 2014). Ainsi, un échantillon raisonné de 100 éleveurs (système pastoral) et agroéleveurs (système agropastoral) a été retenu, en respectant les proportions recensées par l'étude précédente de Senoussi et al. (2014). Le tableau 1 présente la répartition de l'échantillon tenant compte du système d'élevage adopté et de
Tableau 1. Échantillon des éleveurs et agroéleveurs interrogés. Table 1. Sample of surveyed breeders and agro-breeders.

\begin{tabular}{lcc}
\hline & \multicolumn{2}{c}{ Systèmes d'élevage } \\
Taille du troupeau & Agroéleveur & Éleveur \\
\hline$\leq 100$ têtes & 17 & 0 \\
$101-200$ têtes & 29 & 6 \\
$201-500$ têtes & 35 & 4 \\
$501-1950$ têtes & 9 & 0 \\
Total & $\mathbf{9 0}$ & $\mathbf{1 0}$ \\
\hline
\end{tabular}

la taille du troupeau. Les éleveurs sont approchés par le biais de personnes qualifiées de «personnes ressources locales », dont le rôle est de faciliter le contact avec les interviewés.

La méthode des enquêtes permet d'approcher la réalité des systèmes d'élevage avec des délais courts (Lhoste, 2001). Les entretiens de terrain ont été réalisés durant deux mois (juillet et août 2018) par la méthode dite semi-directive décrite par Dockès et Kling-Eveillard (2007). L'objectif était de fournir des informations ayant trait aux différentes ressources alimentaires exploitées pendant la campagne agricole 2017-2018.

\subsection{Présentation de la région d'étude}

La région de M'Sila a été retenue au regard de ses potentialités agropastorales importantes (Fig. 1). Cette région comprend tous les étages bioclimatiques steppiques: semiaride inférieur, aride supérieur et aride inférieur. Cela constitue un atout pour la diversité biogéographique des systèmes d'élevage ovin à étudier. M'Sila a connu une extension des superficies irriguées, surtout pour les cultures fourragères; les superficies fourrgères ont quintuplé grâce à l'application de multiples programmes de mise en valeur des terres agricoles initiés à partir de l'année 2000, pour atteindre 40000 ha en 2018 (DSA, 2018). Le cheptel ovin a également connu un accroissement important pendant la même période; il est passé de 1 million de têtes en 2000 à 1,65 million en 2018, enregistrant un taux moyen de croissance annuel de l'ordre de 3,34\% (DSA, 2018). Ainsi, la région présente un potentiel important de production de viande ovine et de moutons d'El Aïd (fête religieuse de sacrifice des moutons).

La région d'étude s'étale sur une vaste superficie estimée à 1,8 million d'hectares, dont 1 million sont des terres réservées aux parcours. Ces dernières ont subi une dégradation très sévère sur $73 \%$ de leur superficie, due principalement à la surcharge animale (HCDS, 2010).

\subsection{Recueil et préparation des données}

$\mathrm{Au}$ cours des entretiens, nous avons pu collecter des informations portant sur:

- les types et les superficies des différentes pâtures;

- les quantités de compléments distribués aux animaux;

- les périodes d'usage dans l'année;

- les effectifs d'animaux possédés pour chaque catégorie animale.

Les deux étapes de calcul sont les suivantes: 

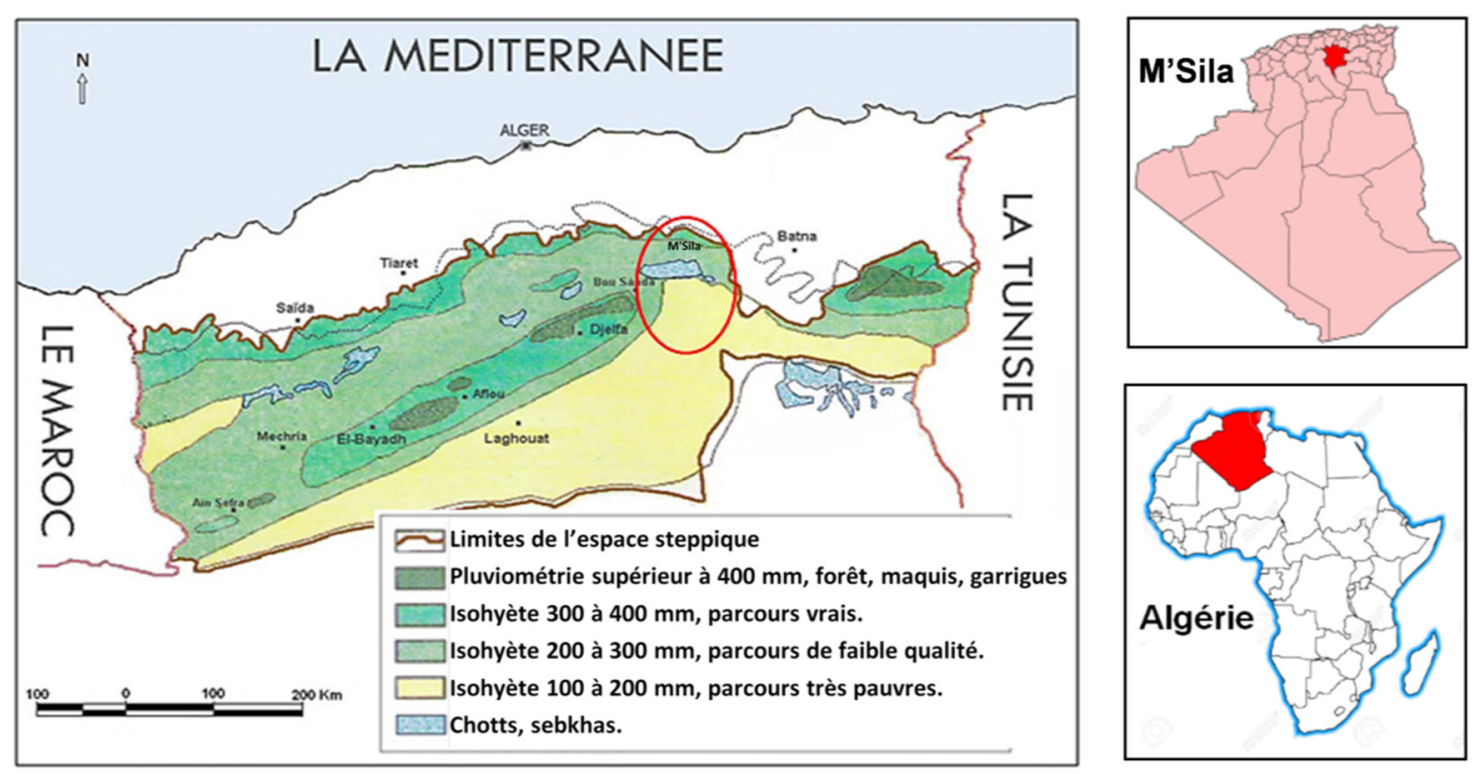

Fig. 1. Localisation de la région d'étude (source du fond de carte: Senoussi et al., 2014).

Fig. 1. Location of the study area (source of the base map: Senoussi et al., 2014).

Tableau 2. Valeur fourragère de référence des ressources alimentaires.

Table 2. Reference forage value of food resources.

\begin{tabular}{|c|c|c|c|}
\hline Type d'aliment & Valeur fourragère (UF/Unité) & Type d'aliment & Valeur fourragère (UF/unité) \\
\hline Son de blé ${ }^{1}$ & $0,74 \mathrm{UF} / \mathrm{kg}$ matière brute & Céréales sinistrées ${ }^{3, * * *}$ & $500 \mathrm{UF} / \mathrm{ha}$ \\
\hline Maïs ${ }^{1}$ & $1,09 \mathrm{UF} / \mathrm{kg}$ matière brute & Paille et foin ${ }^{3}$ & $0,33 \mathrm{UF} / \mathrm{kg}, 25 \mathrm{~kg} /$ botte \\
\hline Orge en vert ${ }^{2, *}$ & $1836 \mathrm{UF} / \mathrm{ha}$ & & \\
\hline Avoine en vert ${ }^{2, *}$ & $3832 \mathrm{UF} / \mathrm{ha}$ & & \\
\hline & $1625 \mathrm{UF} / \mathrm{ha} 3^{\mathrm{e}}$ cycle & & \\
\hline & 1099 UF/ha $4^{\mathrm{e}}$ cycle & & \\
\hline
\end{tabular}

${ }^{1}$ INRA, 2018 (nos calculs) ; ${ }^{2}$ Kerbaa, $1980 ;{ }^{3}$ CIZ/SYFEL, $2004 ; *$ Exploité en déprimage par pâturage sur la parcelle ; ** Exploité en quatre cycles par pâturage sur la parcelle; *** Céréales cultivées sans irrigation et pâturées quand les rendements sont faibles.

- évaluation des apports alimentaires : pour ce faire, nous avons eu recours aux valeurs fourragères de référence disponibles dans la bibliographie (Tab. 2). Nous avons multiplié chaque quantité déclarée exploitée de chaque aliment par la valeur fourragère unitaire correspondante. L'appréciation de la part de la végétation pâturée des parcours étant délicate, nous l'avons donc estimée par soustraction (la différence entre le total des besoins des animaux et la contribution de l'ensemble des autres ressources alimentaires). Ensuite, nous avons pris en compte la distribution de la pluviométrie enregistrée dans la répartition de la contribution des parcours pendant l'année, en multipliant cette dernière par des coefficients calculés majorant les mois pluvieux et minorant les mois secs. De plus, les discussions auprès des éleveurs nous ont permis de considérer comme pertinente l'hypothèse que les besoins des animaux sont généralement satisfaits au vu de leur état;

- estimation des besoins animaux: cette estimation a été effectuée grâce aux coefficients de conversion proposés par Moskal (1983). Il s'agit de coefficients adaptés aux caractéristiques zootechniques du cheptel algérien. Par ailleurs, une majoration a été considérée, liée aux dépenses occasionnées par les déplacements des animaux au pâturage. Cette majoration est de l'ordre de $+50 \%$ pour les troupeaux utilisant des parcours (longues distances parcourues) et de $+20 \%$ pour les troupeaux conduits sans pâturage sur parcours (courtes distances parcourues) (Cirad, 2002). 


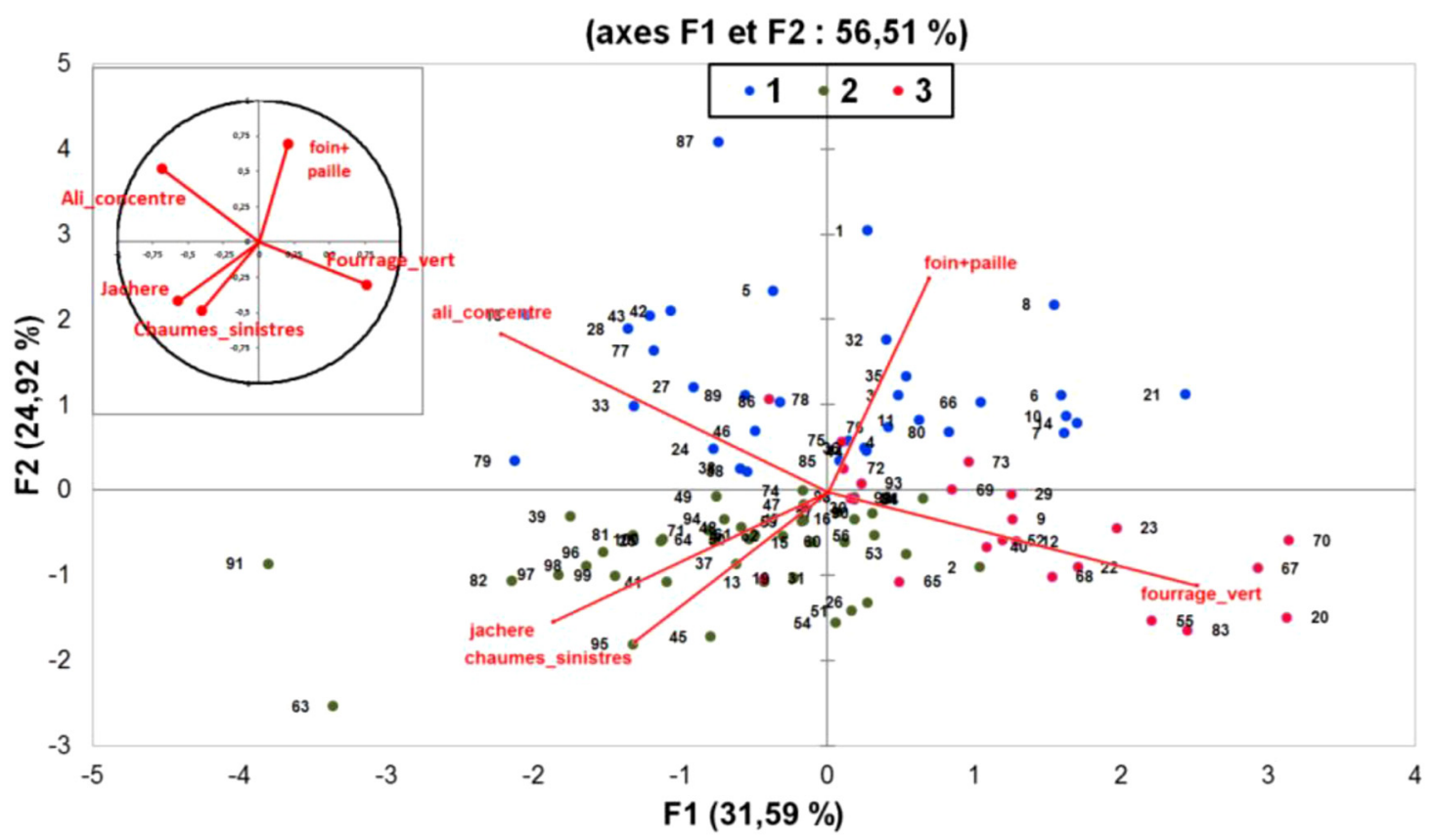

Fig. 2. Représentation des variables et des individus sur les axes F1 et F2 de l'ACP.

Fig. 2. Representation of variables and individuals on axes F1 and F2 of the PCA.

\subsection{Traitements des données}

Les précédentes opérations d'estimation ont permis de constituer un tableau de la composition alimentaire annuelle pour chaque éleveur questionné. En première étape, les contributions alimentaires sont exprimées en UF avant de les considérer en pourcentages rapportés aux besoins des troupeaux, afin de faciliter la lecture et d'éliminer l'écart entre les individus lié à la taille du troupeau.

Par ailleurs, les variables retenues pour l'analyse statistique correspondent aux contributions alimentaires des ressources suivantes :

- les aliments concentrés «ali_concentre»;

- les fourrages verts «fourrage_vert»;

- les chaumes et les céréales sinistrées «chaumes + sinistres $\gg$;

- le foin et la paille «foin + paille»;

- les jachères «jachere».

Il est à signaler que la variable correspondant à la contribution des parcours n'est pas retenue dans l'analyse statistique multivariée, car elle relève d'un calcul entre variables.

Une analyse en composantes principales (ACP) a été réalisée sur les données de la composition alimentaire. L'ACP est une méthode d'analyse multivariée appliquée sur des données quantitatives, dans le but de visualiser la projection des individus et des variables sur les axes factoriels de variations, ainsi que les corrélations entre les variables. Une classification des éleveurs basée sur la similarité de la composition alimentaire était nécessaire pour faciliter l'interprétation des résultats. Cette classification a été possible avec une classification ascendante hiérarchique $(\mathrm{CAH})$, à partir des données de la composition alimentaire.

Pour réaliser une représentation graphique de la composition alimentaire des classes issues des analyses précédentes, nous avons procédé en deux étapes :

- établir les calendriers alimentaires pour chaque classe d'éleveurs, en se basant sur les périodes d'usage déclarées de façon répétée par les éleveurs;

- ensuite, affecter les contributions en UF des aliments de chaque mois pour l'ensemble des éleveurs de chaque classe.

De plus, des tests ANOVA, suivis par un test de Fisher de comparaison par paires, ont été opérés pour comparer les différents paramètres des classes issus des analyses précédentes.

\section{Résultats}

\subsection{Classification des éleveurs selon la composition de l'alimentation du troupeau}

La projection factorielle des vecteurs F1 et F2 de l'ACP résume $56,51 \%$ de la variabilité(Fig. 2). Cette projection cumule le maximum possible de variabilité et semble être la projection la plus compréhensible parmi les combinaisons possibles des vecteurs. Elle montre que les variables «ali concentre» et «fourrage_vert» sont négativement corrélées. Autrement dit, l'utilisation des fourrages verts se traduit par un moindre usage des aliments concentrés et vice-versa.

La CAH fournit une classification des éleveurs en trois classes, basée sur la similitude de la composition alimentaire (C1 : 34 individus, C2 : 42 individus, C3: 24 individus). 


\section{Classe 1}

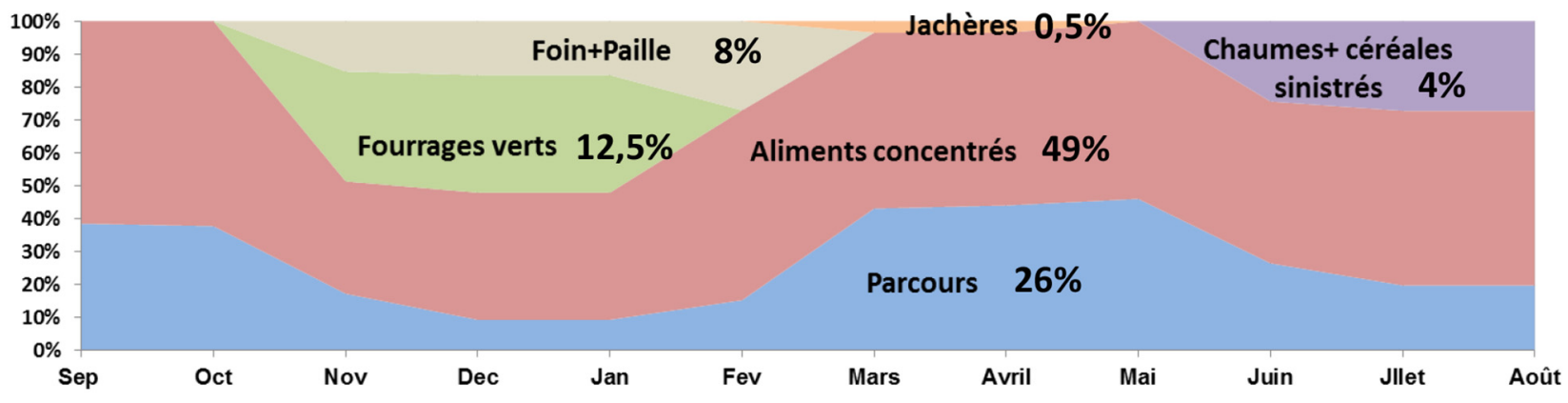

Classe 2

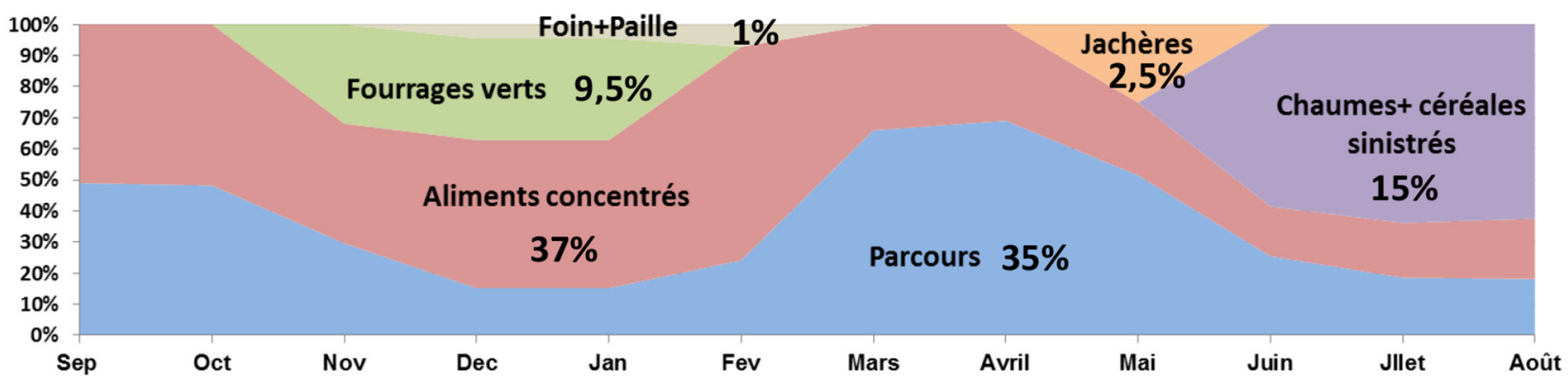

\section{Classe 3}

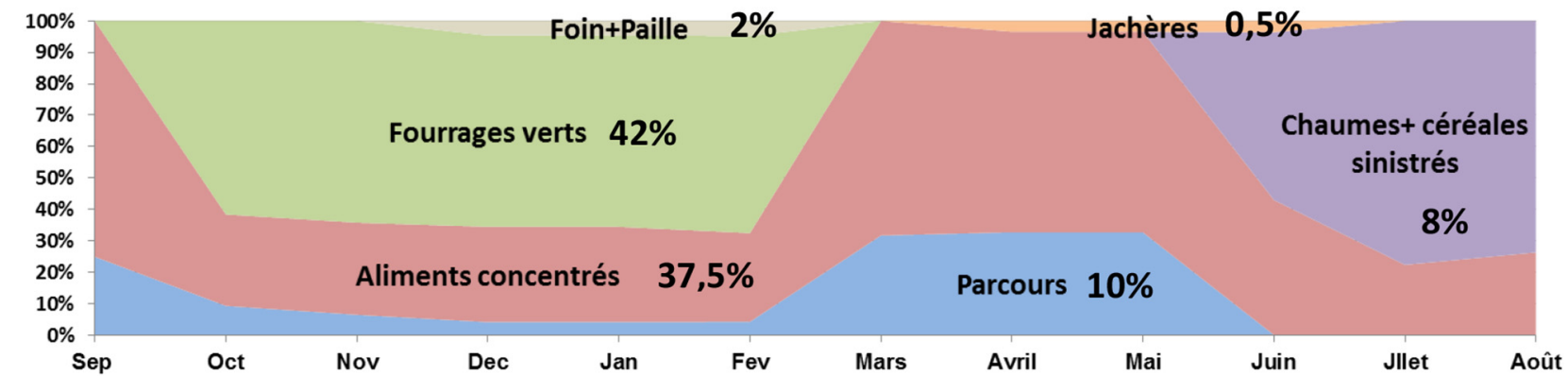

\begin{tabular}{|c|c|c|c|c|c|c|c|c|c|c|c|c|}
\hline (*) & Sep & Oct & Nov & Dec & Jan & Fev & Mars & Avril & Mai & Juin & Jllet & Août \\
\hline $\mathrm{mm}$ & 2 & 14 & 6 & 0 & 8 & 6 & $\mathbf{2 0}$ & $\mathbf{3 6}$ & $\mathbf{2 3}$ & 1 & 0 & 17 \\
\hline
\end{tabular}

* Données de pluviométrie 2017/2018 de la station de Boussaâda au centre de la région d'étude. Source : www.infoclimat.fr

Fig. 3. Représentation graphique des profils alimentaires (Infoclimat, 2019).

Fig. 3. Graphical representation of dietary profiles.

La combinaison des résultats des deux analyses statistiques (ACP et $\mathrm{CAH}$ ) montre une distinction entre classes d'éleveurs proposées par la CAH sur la représentation de l'ACP, et une projection des individus suivant les variables qui les caractérisent: les individus de la classe 1 se retrouvent dans une sphère dominée par les variables "ali_concentre» et «foin + paille»; par contre, les individus de la classe 2 se regroupent autour des vecteurs des variables «chaumes + sinistres» et "jachere». Les individus de la classe 3, quant à eux, semblent être déterminés par la variable «fourrage_vert».

\subsection{Description des profils alimentaires}

La représentation graphique des compositions alimentaires permet de décrire des profils alimentaires correspondant aux trois classes d'éleveurs proposées précédemment (Fig. 3). Ces profils représentent dans les faits trois modalités d'alimentation des troupeaux.

Pour l'ensemble des classes, l'utilisation des ressources alimentaires fluctue dans l'année selon leur disponibilité. D'une part, les ressources alimentaires pâturables (parcours, fourrages verts, chaumes, céréales sinistrées et jachères) ont 
Tableau 3. Principaux paramètres des classes d'éleveurs.

Table 3. Main parameters of breeder classes.

\begin{tabular}{|c|c|c|c|c|c|}
\hline Classe & $\begin{array}{l}\text { Nombre } \\
\text { d'éleveurs }\end{array}$ & $\begin{array}{l}\text { Taille moyenne } \\
\text { de troupeau } \\
\text { (têtes) }\end{array}$ & $\begin{array}{l}\text { Superficie agricole } \\
\text { totale moyenne } \\
\text { (ha) }\end{array}$ & $\begin{array}{l}\text { Superficie moyenne } \\
\text { des cultures } \\
\text { fourragères (ha) }\end{array}$ & $\begin{array}{l}\text { Superficie } \\
\text { moyenne des } \\
\text { cultures fourragères } \\
\text { destinées aux ovins (ha) }\end{array}$ \\
\hline $\mathrm{C} 1$ & 34 & 211 & 11,26 & 6,35 & 3,89 \\
\hline $\mathrm{C} 2$ & 42 & 390 & 54,46 & 7,38 & 6,04 \\
\hline C3 & 24 & 284 & 35,48 & 18,47 & 11,32 \\
\hline
\end{tabular}

une disponibilité saisonnière. D'autre part, les aliments concentrés et les fourrages secs (foin et paille) peuvent être distribués tout au long de l'année selon les besoins. Les fluctuations saisonnières présentées ici sont celles de l'année de l'étude (2017/2018), mais révèlent les grandes tendances de disponibilité et d'usage de chacune des ressources.

Globalement, pour les trois modalités alimentaires, la plus grande part de la ration des animaux (plus de $80 \%$ ) est couverte par trois types de ressources alimentaires: les aliments concentrés, les parcours et les fourrages verts. Pour les éleveurs de la classe 1 , les aliments concentrés assurent la moitié (49\%) de la ration des troupeaux. Pour les éleveurs de la classe 2 , les parcours et les aliments concentrés assurent à part quasi égale $72 \%$ de la ration. Pour les éleveurs de la classe 3, les fourrages verts et les aliments concentrés assurent environ $80 \%$ des besoins des troupeaux.

Les fourrages verts occupent une place importante dans la ration pour les éleveurs de la classe 3 . Ils couvrent $42 \%$ de la ration et assurent une grande partie des besoins des troupeaux pendant la période hivernale. Les chaumes et les céréales sinistrées, malgré leur contribution relativement faible pour les éleveurs des classes 2 et 3 , couvrent plus de la moitié des besoins des animaux pendant la période estivale. Ainsi, nous pouvons identifier des périodes clés pour chaque ressource alimentaire.

\subsection{Les déterminants des profils alimentaires}

Deux paramètres liés aux systèmes d'exploitation sont susceptibles d'influencer le profil alimentaire des troupeaux, à savoir: la taille du troupeau et la pratique de l'agriculture (particulièrement les cultures fourragères). Le tableau 3 récapitule les principaux paramètres des classes d'éleveurs concernant la taille moyenne des troupeaux et la part des cultures fourragères dans la sole agricole.

La taille des troupeaux déclarée va de 50 à 1950 têtes pour l'ensemble des éleveurs et des agroéleveurs interviewés. Une analyse comparative réalisée grâce à l'analyse de variance (ANOVA) a montré qu'il n'existe pas de différence significative entre les trois classes du point de vue de la taille du troupeau. Cela signifie que l'importance du troupeau n'a pas d'influence significative sur la composition de son alimentation.

Concernant la pratique de l'agriculture, $90 \%$ des interviewés exploitent des terres agricoles en plus de l'activité d'élevage. Il s'agit plutôt d'agroéleveurs qui combinent l'élevage et l'agriculture dans la conduite de l'exploitation (systèmes agropastoraux).

La comparaison des superficies en cultures fourragères entre les trois classes a été réalisée par l'ANOVA, suivie du test de Fisher de comparaison des classes deux à deux. Ils ont montré l'existence d'une différence significative entre la classe 3 et les classes 1 et 2 . Cela apparaît cohérent, car les agroéleveurs de la classe 3 présentent un profil alimentaire avec une utilisation plus importante de fourrages verts. Autrement dit, les agroéleveurs de la classe 3 consacrent davantage de superficies aux cultures fourragères pour subvenir aux besoins de leurs troupeaux.

Plusieurs spéculations agricoles sont pratiquées: céréaliculture, cultures fourragères, cultures maraîchères et arboriculture fruitière. Cependant, les cultures liées directement à la conduite alimentaire des troupeaux ovins sont l'orge, l'avoine et la luzerne, constituant ainsi la base de multiples ressources alimentaires : fourrages verts, aliments concentrés, chaumes, céréales sinistrées, foin, et paille.

Pour l'ensemble de l'échantillon, l'orge semble la culture dominante du point de vue des superficies. Elle occupe $72 \%$ de la sole agricole exploitée, dont $17 \%$ en mode irrigué, et $55 \%$ sans irrigation (en sec). L'avoine et la luzerne, conduites seulement en mode irrigué, occupent respectivement $8 \%$ et $2 \%$ de la superficie. Par ailleurs, la contribution des cultures dans les fourrages verts serait respectivement de l'ordre de $80 \%$ pour l'orge, $16 \%$ pour l'avoine, et $4 \%$ pour la luzerne.

\section{Discussion}

L'étude s'est focalisée sur une année jugée moyenne; elle met en évidence les tendances alimentaires actuelles, dont certaines sont inédites. Cela confirme notre hypothèse, qui est que les éleveurs introduisent de nouvelles ressources alimentaires, et cela notamment par l'incorporation de cultures fourragères. Ce choix ne caractérise pas seulement l'année de l'étude, mais révèle plutôt une tendance continue vu l'instabilité inter-annuelle de la pluviométrie. Cette variabilité inter-annuelle a été remarquée, avec des séquences sèches et pluvieuses non périodiques, dans quatre stations des régions arides algériennes, dont une à Djelfa (distant de $100 \mathrm{~km}$ de la région d'étude) (Hirche et al., 2007). Pour l'année de l'étude (2017-2018), bien que le total des précipitations enregistrées durant l'année soit relativement faible $(133 \mathrm{~mm})$, ces précipitations sont considérées comme moyennes par $80 \%$ des interviewés, en référence aux pluies printanières qui 
ont favorisé la repousse de la végétation spontanée des parcours.

De ce fait, la nouvelle tendance à recourir aux cultures fourragères est devenue une pratique régulière pour réduire l'utilisation des aliments concentrés pendant la période hivernale, où les parcours sont peu productifs. Des tendances similaires ont été rapportées dans l'étude de Bencherif (2018), entreprise dans une grande région de la steppe centrale et de l'Ouest. Dans notre zone d'étude, la région de M'Sila, le développement des cultures fourragères a débuté l'année 2000 avec la mise en place du Plan national de développement agricole (PNDA), relatif à la mise en valeur des terres agricoles, notamment avec l'introduction de systèmes d'irrigation de pointe. L'extension des superficies fourragères (40000 ha en 2018) témoigne de la tendance à utiliser ces ressources alimentaires. Il s'agit certainement d'un passage vers l'agropastoralisme, en tant que stratégie « anti-aléatoire» permettant de mieux maîtriser les incertitudes climatiques et économiques (Bourbouze, 2000). Cet agropastoralisme est qualifié de mode de conduite raisonné, intégrant de nouvelles ressources alimentaires plus stables, moins chères et plus productives.

Les cultures fourragères utilisées sont principalement l'orge, puis à un moindre degré l'avoine, suivie de la luzerne. Cette répartition s'explique par des éléments pratiques : l'orge demeure la culture dominante à cause de ses multiples modes d'utilisation dans différentes circonstances climatiques: déprimage comme fourrage vert en période hivernale, aliment concentré et sous-produits après la récolte (orge en grain, chaumes et paille), ou pâturée en entier si les rendements sont faibles (orge sinistrée). Abdelguerfi et al. (2008) ont expliqué l'intérêt porté à la culture de l'orge par rapport à celle de l'avoine et de la luzerne:

- l'avoine est moins répandue en milieu steppique, car elle est plutôt considérée comme un fourrage à foin et son extension est subordonnée au prix de la semence sur le marché ;

- la luzerne, d'introduction récente depuis dix ans à peine, commence juste à prendre place dans l'alimentation des ovins. Actuellement, elle est très sollicitée vu son avantage productif. La luzerne était proposée comme fourrage de remplacement des cultures classiques dans les périmètres irrigués (Abdelguerfi et al., 2008); néanmoins, la cherté et la non disponibilité des semences constituent une entrave à son extension.

La complémentation du reste des besoins par la végétation spontanée (fourrages naturels) des parcours traduit une réelle logique de surexploitation des espaces pastoraux de la part des éleveurs. La surexploitation des parcours steppiques a été dénoncée par plusieurs études, à l'instar de celles menées par Aïdoud et al. (2006), Bencherif (2018), Bensouiah (2004), Nedjraoui et Bédrani (2008). Ainsi, bien que les nouvelles alternatives alimentaires garantissent une ressource non négligeable, elles se développent dans une logique d'improvisation individuelle qui n'intègre aucun intérêt en terme de gestion rationnelle des ressources naturelles, principalement les parcours.

\section{Conclusion}

Du fait du manque de fourrages naturels des parcours et face aux instabilités liées à l'approvisionnement en aliments concentrés, les éleveurs de la région de M'Sila montrent une grande diversité d'usage des différentes ressources alimentaires disponibles et expérimentent de nouvelles pistes d'affouragement. Les nouvelles tendances s'articulent autour de l'intégration des cultures fourragères dans la conduite alimentaire des ovins. Ces tendances alimentaires apparaissent comme des solutions curatives incomplètes, car elles manquent de maîtrise technique. Cependant, elles peuvent inspirer des pistes de réflexions sur des solutions possibles au manque de ressources fourragères en milieu steppique, en réduisant la charge animale sur les parcours à certaines périodes. Néanmoins, d'autres recherches s'avèrent nécessaires pour évaluer la faisabilité de ces alternatives alimentaires, les améliorer et les intégrer dans un processus de développement durable de la région.

\section{Références}

Abbas K. 2004. Dégradation des parcours steppiques. Pour une lecture différente du rôle de l'élevage. Fourrages 180: 541-556.

Abdelguerfi A, Laouar M, M'Hammedi Bouzina M. 2008. Les productions fourragères et pastorales en Algérie : situation et possibilités d'amélioration. Agriculture \& développement 6: 14-25.

Aïdoud A, Le Floc'h É, Le Houérou HN. 2006. Les steppes arides du nord de l'Afrique. Science et changements planétaires/Sécheresse 17(1-2): 19-30.

Bencherif S. 2018. Origines et transformations récentes de l'élevage pastoral de la steppe algérienne. Revue internationale des études $d u$ développement 236(4): 55-79.

Bensouiah R. 2004. Pasteurs et agropasteurs de la steppe algérienne : enquête sur la région de Djebel Amour. Strates - Matériaux pour la recherche en sciences sociales (11): $13 \mathrm{p}$.

Bourbouze A. 2000. Pastoralisme au Maghreb : la révolution silencieuse. Fourrages 161: 3-21.

Cirad. 2002. Mémento de l'agronome. Paris (Caillavet) : Cirad-Gret, Ministère des affaires étrangères, $1694 \mathrm{p}$.

CIZ/SYFEL. 2004. Circuit d'information zootechnique. ITELV, BabaAli, document interne. In : Merdjane L, Yakhlef H. 2016. Le déficit fourrager en zone semi-aride : une contrainte récurrente au développement durable de l'élevage des ruminants. Revue agriculture 1: 43-51.

Dockès AC, Kling-Eveillard F. 2007. Les représentations de l'animal et du bien-être animal par les éleveurs français. INRA Prod Anim 20 (1): 23-28.

DSA. 2018. Statistiques agricoles. Algérie : Direction des services agricoles de M'Sila.

FAO. 2017. Données statistiques du cheptel ovin 1961-2017. Bases statistiques en ligne FAOSTAT, mise à jour le : 20 décembre 2018, [8 octobre 2019]. http://www.fao.org/faostat/fr/

HCDS. 2010. Les potentialités agropastorales de la steppe algérienne : requêtes cartographiques, analyse et interprétation de l'information géographique. Djelfa, Algérie: HCDS et BNEDER, 80 p.

Hirche A, Boughani A, Salamani M. 2007. Évolution de la pluviosité annuelle dans quelques stations arides algériennes. Science et changements planétaires/Sécheresse 18(4): 314-320. 
Infoclimat. Climatologie de l'année 2017-2018 à Bou Saâda. [2019/ 11/05]. https://www.infoclimat.fr/climatologie/annee/2017/bousaada/valeurs/60515.html; https://www.infoclimat.fr/climatologie/ annee/2018/bou-saada/valeurs/60515.html

INRA. 2018. Alimentation des ruminants. Versailles, France: Éditions Quae, 728 p.

Kerbaa F. 1980. Guide de la valeur alimentaire des fourrages cultivés en Algérie. Alger, Algérie: ITELV, 17 p.

Lhoste P. 2001. L'étude et le diagnostic des systèmes d'élevage. Atelier de formation des agronomes SCV, Madagascar: 1-32.
Moskal S. 1983. Essai d'évaluation du bilan fourrager en Algérie du Nord. Alger, Algérie: INA, 25 p.

Nedjraoui D, Bédrani S. 2008. La désertification dans les steppes algériennes : causes, impacts et actions de lutte. VertigO 8(1): 15 p.

Senoussi A, Chehma A, Bensemaoune Y. 2011. La steppe algérienne à l'aube du $\mathrm{III}^{\mathrm{e}}$ millénaire : quel devenir? Annales des sciences et technologie (AST) 3(2): 129-138.

Senoussi A, Hadbaoui I, Huguenin J. 2014. L'espace pastoral dans la région de M'sila, Algérie : état et perspectives de réhabilitation. Livestock Research for Rural Development 26(11): 7 p.

Citation de l'article : Hadbaoui I, Senoussi A, Huguenin J. 2020. Les modalités d'alimentation des troupeaux ovins en steppe algérienne, région de M'Sila: pratiques et tendances. Cah. Agric. 29: 28. 